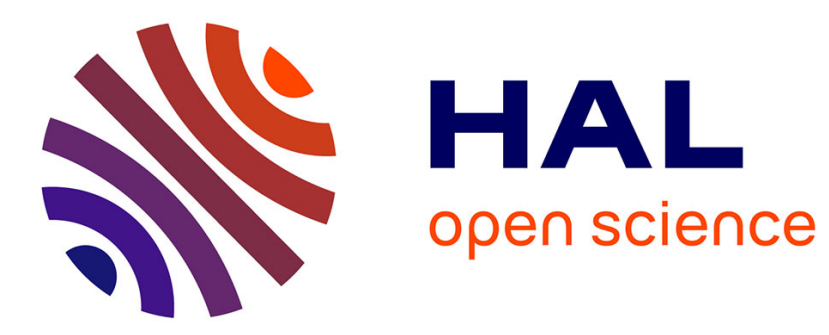

\title{
Correlations in single photon amplification: stimulated versus spontaneous processes
}

\author{
A. Heidmann, S. Reynaud
}

\section{To cite this version:}

A. Heidmann, S. Reynaud. Correlations in single photon amplification: stimulated versus spontaneous processes. Journal de Physique, 1984, 45 (5), pp.873-883. 10.1051/jphys:01984004505087300 . jpa00209820

\section{HAL Id: jpa-00209820 https://hal.science/jpa-00209820}

Submitted on 1 Jan 1984

HAL is a multi-disciplinary open access archive for the deposit and dissemination of scientific research documents, whether they are published or not. The documents may come from teaching and research institutions in France or abroad, or from public or private research centers.
L'archive ouverte pluridisciplinaire HAL, est destinée au dépôt et à la diffusion de documents scientifiques de niveau recherche, publiés ou non, émanant des établissements d'enseignement et de recherche français ou étrangers, des laboratoires publics ou privés. 
Classification

Physics Abstracts

$42.50-03.65-32.80$

\title{
Correlations in single photon amplification : stimulated versus spontaneous processes
}

\author{
A. Heidmann and S. Reynaud \\ Laboratoire de Spectroscopie Hertzienne de l'E.N.S., 24, rue Lhomond, 75231 Paris Cedex 05, France
}

(Reçu le 16 novembre 1983, accepté le 3 janvier 1984)

\begin{abstract}
Résumé. - On étudie les corrélations temporelles entre photons sortant d'un amplificateur optique (amplification par émission stimulée) lorsque l'état entrant comprend un seul photon. On montre que cet amplificateur n'apparaît alors pas du tout comme un «duplicateur de photons " mais plutôt comme une source de bruit statistiquement indépendante du champ entrant (bruit d'émission spontanée).
\end{abstract}

\begin{abstract}
We study temporal correlations between photons going out an optical amplifier (stimulated emission amplification) when the input consists of a single photon. It is shown that this amplifier thus appears not at all as a " photon cloner " but rather as a noise source statistically independent from the input field (spontaneous emission noise).
\end{abstract}

\section{Introduction.}

Amplification of light by stimulated emission is the basis of laser action. So the statistical properties of such an optical amplification have been extensively investigated during the last twenty years [1-11]. More recently, it has been pointed out that novel questions arise when single photon optical amplification is considered [12-13]. These questions can be illustrated by the experiment sketched on figure 1 : temporal correlations are studied on the output of an optical amplifier when the input consists of a single photon (how such an experiment could be realized will be discussed later).

It might be expected that stimulated emission provides a pair of correlated photons which can be discriminated from spontaneous ones. If it was the case, it would be possible to use stimulated emission in order to "clone" ("duplicate») photons. But stimulated emission is known to produce two photons having equal frequencies and the existence of temporal correlations between them seems incompatible with the dispersion inequality $\Delta \omega \Delta t>1$. On the other hand, it must be noted that intensity correlations can be detected on the light emitted by two statistically independent sources (Hanbury-Brown and Twiss effect [14-18]). Spontaneously emitted photons may therefore appear correlated with the input photon. In order to conclude to the possibility of the impossibility of cloning photons, it seems necessary to

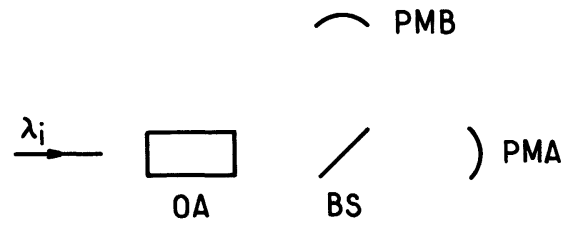

Fig. 1. - A single input photon (input mode $\lambda_{\mathbf{i}}$ ) comes on an optical amplifier OA. Temporal correlations between photons going out the amplifier are studied using two photomultipliers PMA and PMB located behind a beam splitter BS.

understand in more details the respective contributions of stimulated and spontaneous emission to the correlation experiment of figure 1 .

Understanding correlations which appear in single photon amplification is also important for the discussion of an experiment proposed by several authors [19-22] which is sketched in figure 2. The optical amplifier is now located in one of the two arms of a Mach-Zender interferometer. A single photon is supposed to enter the interferometer. Temporal correlations are studied using two detectors located on one hand (detector A) behind the beam splitter which is in the same arm as the amplifier and on the other hand (detector B) at one of the outputs of the interferometer. If the amplifier could be considered as an ideal « duplicator " (i.e. providing two output photons for a single 


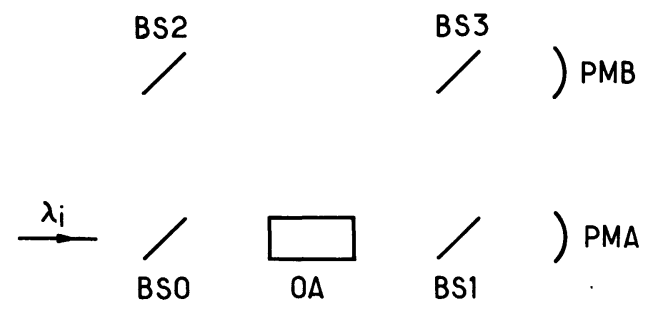

Fig. 2. - A single input photon (input mode $\lambda_{\mathrm{i}}$ ) comes on the input beam splitter BS0 of a Mach-Zender interferometer. An optical amplifier OA and a beam splitter BS1 are placed in the arm 1 of the interferometer and a beam splitter BS2 in the arm 2. Correlations are studied using a photomultiplier PMA located behind BS1 and a photomultiplier PMB located at one of the output of the interferometer (BS3 output beam splitter).

input photon but no output photon in absence of input photon), then the detection of a photon at $\mathrm{A}$ would imply that the photon entering the interferometer has passed through arm 1 . So, interference would be destroyed at $B$. This is no longer true when one takes into account photons spontaneously emitted by the amplifier [13, 22-24]. As the experiment of figure 2 has been considered as challenging quantum mechanics [19-21], it is particularly important to know exactly what are the quantum predictions for it.

Some interesting results have already been obtained when the optical amplifier can be treated as a linear gain tube [23-24]. We will choose in this paper another model of optical amplifier : a two level atom (atomic frequency $\omega_{0}$ ) irradiated by a nearly resonant laser (frequency $\omega_{\mathrm{L}}$ ). Such a system is known to be amplifying at some frequencies [25-26]. This amplification can be understood as resulting from a population inversion on a transition of the compound system " atom plus laser photons interacting together", i.e. the "dressed atom" [27-29]. For a non saturating laser, amplification occurs at frequency $2 \omega_{\mathrm{L}}-\omega_{0}$, due to the non linear process sketched on figure 3 : absorption of two laser photons and emission of two photons having frequencies close to $2 \omega_{\mathrm{L}}-\omega_{0}$ and $\omega_{0}$ [30-32].

We will in this paper derive the quantum expressions for the correlation signals corresponding to the two experiments of figure 1 and figure 2 when optical amplification is produced by the process of figure 3 . We first show that the correlation signal of the first experiment (Fig. 1) can be expressed in terms of transition amplitudes describing the emission of photons by the amplifier and their destruction by the detectors $(\S 2)$. We then compare the amplitudes associated with the spontaneous and stimulated processes $(\S 3)$ and give a diagrammatic representation of these amplitudes $(\$ 4)$. We calculate explicitly the delay dependence of the correlation signal $(\$ 5)$. We finally calculate the correlation signal corresponding to the experiment of figure $2(\$ 7)$.
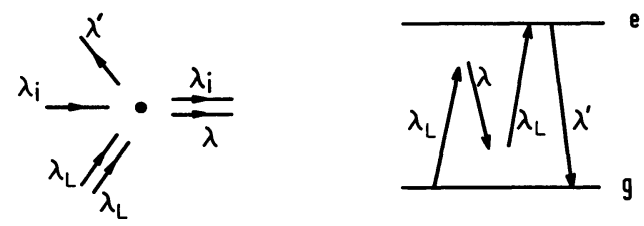

Fig. 3. - Optical amplifier studied in this paper : two level atom irradiated by a nearly resonant laser. Amplification occurs due to the non linear process where the atom absorbs two laser photons $\lambda_{\mathrm{L}}$ and emit two photons $\lambda$ and $\lambda^{\prime}$. Emission of the $\lambda$ photon can be stimulated by the input photon (when $\lambda=\lambda_{\mathrm{i}}$ ) or spontaneous (when $\lambda \neq \lambda_{\mathrm{i}}$ ).

Two other points are discussed in the paper. First we discuss what conditions are required in order to be sure that the experiment of figure 1 concerns effectively single photon amplification (same conditions are valid in the case of figure 2). In other words, what conditions are required for the " one input photon " contribution to be predominant $(\S 6)$. Finally, we derive an inequality for the fringe contrast in the interference experiment of figure 2 , which is valid whatever the design of the optical amplifier may be $(\S 8)$.

\section{Interpretation of the correlation signal in terms of transition amplitudes.}

We want to show in this section that the correlation signal corresponding to the experiment sketched on figure 1 can be expressed in terms of transition amplitudes describing the emission of photons by the amplifier and their destruction by the detectors. We will closely follow the treatment developed in [33] for the study of correlation signals in resonance fluorescence.

The emission of photons by the amplifier is treated by scattering theory. The "precollision " wave packet $\left|\psi_{\mathrm{i}}\right\rangle$ describes the emitting atom in its ground state $g$ in presence of $N$ laser photons (laser mode $\lambda_{\mathrm{L}}$ ) and one « input » photon (input mode $\lambda_{\mathrm{i}}$ ):

$$
\left|\psi_{\mathrm{i}}\right\rangle=\left|g, N \lambda_{\mathrm{L}}, \lambda_{\mathrm{i}}\right\rangle \text {. }
$$

Actually, the laser field is described more realistically by a quasiclassical superposition of Fock states (for example, a coherent state). But its representation by a pure Fock state $\left|N \lambda_{L}\right\rangle$ makes no difference in the forthcoming discussions while appreciably simplifying the notations.

The " postcollision " wave packet $\left|\psi_{\mathrm{f}}\right\rangle$ is obtained by action of the $S$ matrix calculated through formal scattering theory [34] :

$$
\left|\psi_{\mathrm{f}}\right\rangle=S\left|\psi_{\mathrm{i}}\right\rangle \text {. }
$$

This postcollision packet can be expanded on the states where the atom is in its ground state in presence of one input photon. $(N-n)$ laser photons and $n$ fluorescence photons (scattering of $n$ laser photons). 
The scattering of the input photon is indeed much less probable than the scattering of laser photons and it can be neglected. For a non saturating laser excitation, such an expansion can be restricted to its first terms [33] :

i) zeroth order $\left(\operatorname{ket}\left|g, N \lambda_{\mathrm{L}}, \lambda_{\mathrm{i}}\right\rangle\right)$;

ii) first order : the scattering of one photon from the laser mode $\lambda_{L}$ to the mode $\lambda$ is described by the appearance of the kets $\left|g,(N-1) \lambda_{\mathrm{L}}, \lambda_{\mathrm{i}}, \lambda\right\rangle$ in the first order expansion of $S\left|\psi_{\mathrm{i}}\right\rangle$. Energy conservation implies that the emitted photon has the same frequency as the laser one ( $\omega_{\lambda}=\omega_{\mathrm{L}}$ : elastic Rayleigh scattering);

iii) second order : the scattering of two laser photons is described by the kets $\left|g,(N-2) \lambda_{\mathrm{L}}, \lambda_{\mathrm{i}}, \lambda, \lambda^{\prime}\right\rangle$. Energy conservation implies $\omega_{\lambda}+\omega_{\lambda^{\prime}}=2 \omega_{\mathrm{L}}$ but not $\omega_{\lambda}=\omega_{\lambda^{\prime}}=\omega_{\mathbf{L}}$ (inelastic scattering). It appears on figure 3 that such a process is resonant when the excited level is populated by the multiphoton process " absorption of two laser photons and emission of a $\omega_{\lambda}$ one ", i.e. when $\left(\omega_{\lambda}-\left(2 \omega_{\mathbf{L}}-\omega_{0}\right)\right)$ and $\left(\omega_{\lambda^{\prime}}-\omega_{0}\right)$ are close to zero (within the radiative linewidth $\Gamma$ ). Second order scattering then explains the two sidebands of the fluorescence triplet [33].

Higher order terms have a much smaller contribution than the previous ones to the correlation signal discussed in this paper and they will be ignored in the following.

Knowing the state $\left|\psi_{\mathrm{f}}\right\rangle$ of the scattered field, we have now to calculate the correlation signal corresponding to figure 1 . It is well known that such a signal can be expressed as a correlation function of the electric field involving the values of the field at the two detectors A and B [35]. In order to simplify the notations, we will relate these fields (transmitted and reflected by the beam splitter) to the electric field $E(r, t)$ which would exist in the absence of the beam splitter. The correlation signal $P$ (more precisely; the joint probability $P$ of two photon detection) can thus be written :

$$
P=C T R\left\langle\psi_{\mathrm{f}}\left|E_{\mathrm{A}}^{-} E_{\mathrm{B}}^{-} E_{\mathrm{B}}^{+} E_{\mathrm{A}}^{+}\right| \psi_{\mathrm{f}}\right\rangle
$$

where $T$ and $R$ are the transmission and reflection coefficients of the beam splitter and $C$ some multiplicative constant. $E_{\mathrm{A}}^{+}$and $E_{\mathrm{A}}^{-}$are respectively the positive and negative frequency components of the electric field $E\left(r_{\mathrm{A}}, t_{\mathrm{A}}\right)$ taken in the Heisenberg point of view for values of the position $r_{\mathrm{A}}$ and time $t_{\mathrm{A}}$ corresponding to the detector $\mathrm{A}$ (the same definitions apply to $E_{\mathrm{B}}^{+}$and $E_{\mathrm{B}}^{-}$but $r_{\mathrm{B}}$ is such that its " image " reflected by the beam splitter corresponds to the actual position of the detector B) :

$$
E_{\mathrm{A}}^{+}=i \sum_{\mu} e_{\mathrm{A}} \cdot \varepsilon_{\mu}\left(r_{\mathrm{A}}\right) g\left(\omega_{\mu}\right) a_{\mu} \mathrm{e}^{-i \omega_{\mu} t_{\mathrm{A}}}
$$

where $e_{\mathrm{A}}$ is the detection polarization and $\boldsymbol{\varepsilon}_{\mu}$ a complete set of orthonormal field distributions, for exam- ple the plane waves of wave vector $k_{\mu}$, frequency $\omega_{\mu}$ and polarization $e_{\mu} ; a_{\mu}$ is the destruction operator of a photon in the mode $\mu$ and $g\left(\omega_{\mu}\right)$ is a filtering amplitude describing the effect of the frequency filters placed in front of the two detectors which select the frequencies close to $\left(2 \omega_{\mathrm{L}}-\omega_{0}\right)$ :

$$
\begin{array}{ll}
g(\omega)=1 \text { when } \quad \omega \simeq\left(2 \omega_{\mathrm{L}}-\omega_{0}\right) \\
g(\omega)=0 \text { when } \omega \simeq \omega_{\mathrm{L}} \text { or } \omega \simeq \omega_{0} .
\end{array}
$$

Note that $P$ can be expressed as the norm of a state vector $|\varphi\rangle$ :

$$
P=C R T\langle\varphi \mid \varphi\rangle
$$

with :

$$
|\varphi\rangle=E_{\mathrm{B}}^{+} E_{\mathrm{A}}^{+}\left|\psi_{\mathrm{f}}\right\rangle=E_{\mathrm{B}}^{+} E_{\mathrm{A}}^{+} S\left|\psi_{\mathrm{i}}\right\rangle .
$$

Now the operator $E_{\mathrm{B}}^{+} E_{\mathrm{A}}^{+}$destroys two photons having frequencies close to $\left(2 \omega_{\mathrm{L}}-\omega_{0}\right)$. It follows that only the second order terms of the perturbative expansion of $S\left|\psi_{\mathrm{i}}\right\rangle$ will contribute to the correlation signal. In other words, $|\varphi\rangle$ can be expanded as :

$$
\begin{array}{r}
|\varphi\rangle=\sum_{\lambda^{\prime}} \sum_{\lambda}\left[E_{\mathrm{B}}^{+} E_{\mathrm{A}}^{+}\left|g,(N-2) \lambda_{\mathrm{L}}, \lambda_{\mathrm{i}}, \lambda, \lambda^{\prime}\right\rangle\right. \\
\left.\left\langle g,(N-2) \lambda_{\mathrm{L}}, \lambda_{\mathrm{i}}, \lambda, \lambda^{\prime}|S| \psi_{\mathrm{i}}\right\rangle\right]
\end{array}
$$

(with $\omega_{\lambda^{\prime}} \simeq \omega_{0}$ and $\omega_{\lambda} \simeq 2 \omega_{\mathrm{L}}-\omega_{0}$ ).

As $E_{\mathrm{B}}^{+} E_{\mathrm{A}}^{+}\left|g,(N-2) \lambda_{\mathrm{L}}, \lambda_{\mathrm{i}}, \lambda, \lambda^{\prime}\right\rangle$ is proportional to $\left|g,(N-2) \lambda_{L}, \lambda^{\prime}\right\rangle$ (destruction of the two photons having frequencies close to $\left.2 \omega_{\mathrm{L}}-\omega_{0}\right),|\varphi\rangle$ can finally be written :

$$
|\varphi\rangle=\sum_{\lambda^{\prime}} \sum_{\lambda}\left|g,(N-2) \lambda_{\mathrm{L}}, \lambda^{\prime}\right\rangle \mathcal{A}\left(\lambda, \lambda^{\prime}\right)
$$

with :

$$
\begin{aligned}
& \mathcal{A}\left(\lambda, \lambda^{\prime}\right)= \\
& \left\langle g,(N-2) \lambda_{\mathrm{L}}, \lambda^{\prime}\left|E_{\mathrm{B}}^{+} E_{\mathrm{A}}^{+}\right| g,(N-2) \lambda_{\mathrm{L}}, \lambda_{\mathrm{i}}, \lambda, \lambda^{\prime}\right\rangle \\
& \quad\left\langle g,(N-2) \lambda_{\mathbf{L}}, \lambda_{\mathrm{i}}, \lambda, \lambda^{\prime}|S| g, N \lambda_{\mathrm{L}}, \lambda_{\mathrm{i}}\right\rangle .
\end{aligned}
$$

The corresponding expression for the correlation signal :

$$
P=C R T \sum_{\lambda^{\prime}}\left|\sum_{\lambda} \mathcal{A}\left(\lambda, \lambda^{\prime}\right)\right|^{2}
$$

has a straightforward interpretation : $\mathcal{A}\left(\lambda, \lambda^{\prime}\right)$ is the transition amplitude from the initial state $\left|g, N \lambda_{\mathbf{L}}, \lambda_{\mathrm{i}}\right\rangle$ to the final one $\left|g,(N-2) \lambda_{\mathrm{L}}, \lambda^{\prime}\right\rangle$ through the emission of two photons $\lambda$ and $\lambda^{\prime}$ by the emitting atom and the destruction of two photons $\lambda$ and $\lambda_{\mathrm{i}}$ by the detectors A and B. To calculate $P$, we have to add the transition amplitudes corresponding to indistinguishable paths (various values of $\lambda$ ) and then to add the transition probabilities corresponding to distinct final states (the $\lambda^{\prime}$ photon is not detected and various values of $\lambda^{\prime}$ are thus distinguished). 
3. Comparison between the spontaneous and stimulated amplitudes.

We will now compare the transition amplitudes $\mathcal{A}\left(\lambda, \lambda^{\prime}\right)$ associated with spontaneous and stimulated emission processes. We have thus to discuss successively the structure of the term $\left\langle g,(N-2) \lambda_{\mathrm{L}}, \lambda_{\mathrm{i}}, \lambda\right.$, $\left.\lambda^{\prime}|S| g, N \lambda_{\mathrm{L}}, \lambda_{\mathrm{i}}\right\rangle$ which describes the scattering of two laser photons and the structure of the term $\left\langle g,(N-2) \lambda_{\mathrm{L}}, \lambda^{\prime}\left|E_{\mathrm{B}}^{+} E_{\mathrm{A}}^{+}\right| g,(N-2) \lambda_{\mathrm{L}}, \lambda_{\mathrm{i}}, \lambda, \lambda^{\prime}\right\rangle$ which corresponds to the detection of the two photons having frequencies close to $\left(2 \omega_{\mathrm{L}}-\omega_{0}\right)$.

We will note $S_{2}\left(\lambda, \lambda^{\prime}\right)$ the scattering amplitude of two laser photons into the modes $\lambda$ and $\lambda^{\prime}$ by an atom irradiated only by $N$ laser photons (in absence of the incident input photon $\lambda_{\mathrm{i}}$ ) :

$$
S_{2}\left(\lambda, \lambda^{\prime}\right)=\left\langle g,(N-2) \lambda_{\mathrm{L}}, \lambda, \lambda^{\prime}|S| g, N \lambda_{\mathrm{L}}\right\rangle
$$

(the structure of this term is discussed in [33]). In presence of an incident input photon, the scattering amplitude is not modified if the emission mode $\lambda$ is different from the input mode $\lambda_{\mathrm{i}}$ :

$$
\begin{array}{r}
\left\langle g,(N-2) \lambda_{\mathrm{L}}, \lambda_{\mathrm{i}}, \lambda, \lambda^{\prime}|S| g, N \lambda_{\mathrm{L}}, \lambda_{\mathrm{i}}\right\rangle= \\
=S_{2}\left(\lambda, \lambda^{\prime}\right) \text { if } \lambda \neq \lambda_{\mathrm{i}}
\end{array}
$$

(the other emission mode $\lambda^{\prime}$ of frequency close to $\omega_{0}$ is always different from $\lambda_{\mathrm{i}}$ ). But the presence of the incident input photon stimulates the scattering processes where a $\lambda_{i}$ photon is emitted. The scattering amplitudes corresponding to such stimulated processes are larger than those corresponding to spontaneous ones by a factor $\sqrt{2}$ :

$$
\begin{aligned}
\left\langle g,(N-2) \lambda_{\mathrm{L}}, 2 \lambda_{\mathrm{i}}, \lambda^{\prime}|S| g,\right. & \left.N \lambda_{\mathrm{L}}, \lambda_{\mathrm{i}}\right\rangle= \\
= & \sqrt{2} S_{2}\left(\lambda_{\mathrm{i}}, \lambda^{\prime}\right) .
\end{aligned}
$$

We consider now the detection amplitudes :

$$
\begin{aligned}
\langle g,(N-2) & \left.\lambda_{\mathrm{L}}, \lambda^{\prime}\left|E_{\mathrm{B}}^{+} E_{\mathrm{A}}^{+}\right| g,(N-2) \lambda_{\mathrm{L}}, \lambda_{\mathrm{i}}, \lambda, \lambda^{\prime}\right\rangle= \\
= & \left\langle 0\left|E_{\mathrm{B}}^{+} E_{\mathrm{A}}^{+}\right| \lambda_{\mathrm{i}}, \lambda\right\rangle \\
& =\sum_{\mu} \sum_{\mu^{\prime}} \alpha_{\mathrm{A}}(\mu) \alpha_{\mathrm{B}}\left(\mu^{\prime}\right)\left\langle 0\left|a_{\mu^{\prime}} a_{\mu}\right| \lambda_{\mathrm{i}}, \lambda\right\rangle
\end{aligned}
$$

where :

$$
\alpha_{\mathrm{A}}(\mu)=i e_{\mathrm{A}} \cdot \boldsymbol{\varepsilon}_{\mu}\left(r_{\mathrm{A}}\right) g\left(\omega_{\mu}\right) \mathrm{e}^{-i \omega_{\mu} t_{\mathrm{A}}}
$$

(same expression for $\alpha_{B}\left(\mu^{\prime}\right)$ : see equation (2.4)). When the two photons $\lambda_{\mathrm{i}}$ and $\lambda$ are distinguishable, there are two couples $\left(\mu, \mu^{\prime}\right)$ which contribute to (3.5) : either $\mu=\lambda_{\mathrm{i}}$ and $\mu^{\prime}=\lambda$ which corresponds to the detection of the $\lambda_{\mathrm{i}}$ photon on the detector $A$ and to the detection of the $\lambda$ one on B or vice-versa. So the detection amplitude can be written :

$$
\begin{aligned}
& \left\langle 0\left|E_{B}^{+} E_{A}^{+}\right| \lambda_{i}, \lambda\right\rangle= \\
& \quad=\alpha_{A}\left(\lambda_{i}\right) \alpha_{B}(\lambda)+\alpha_{A}(\lambda) \alpha_{B}\left(\lambda_{i}\right) \text { if } \lambda \neq \lambda_{i} .
\end{aligned}
$$

When $\lambda_{\mathrm{i}}$ and $\lambda$ are equal, there is only one possibility $\mu=\mu^{\prime}=\lambda_{\mathrm{i}}=\lambda$ but the first detection is stimulated by the presence of the second photon :

$$
\left\langle 0\left|E_{\mathrm{B}}^{+} E_{\mathrm{A}}^{+}\right| 2 \lambda_{\mathrm{i}}\right\rangle=\sqrt{2} \alpha_{\mathrm{A}}\left(\lambda_{\mathrm{i}}\right) \alpha_{\mathrm{B}}\left(\lambda_{\mathrm{i}}\right) .
$$

We get then the transition amplitudes $\mathcal{A}\left(\lambda, \lambda^{\prime}\right)$ by multiplying the scattering amplitudes and the detection ones. In the cases of the spontaneous amplitudes $\left(\lambda \neq \lambda_{\mathrm{i}}\right)$, equations (3.2) and (3.7) give :

$$
\mathcal{A}\left(\lambda, \lambda^{\prime}\right)=S_{2}\left(\lambda, \lambda^{\prime}\right)\left[\alpha_{\mathbf{A}}\left(\lambda_{\mathbf{i}}\right) \alpha_{\mathbf{B}}(\lambda)+\alpha_{\mathbf{A}}(\lambda) \alpha_{\mathbf{B}}\left(\lambda_{\mathrm{i}}\right)\right] .
$$

For stimulated amplitudes $\left(\lambda=\lambda_{\mathrm{i}}\right)$, equations (3.3) and (3.8) lead to :

$$
\begin{aligned}
& \mathcal{A}\left(\lambda_{\mathrm{i}}, \lambda^{\prime}\right)=\sqrt{2} S_{2}\left(\lambda_{\mathrm{i}}, \lambda^{\prime}\right) \sqrt{2} \alpha_{\mathbf{A}}\left(\lambda_{\mathrm{i}}\right) \alpha_{\mathbf{B}}\left(\lambda_{\mathrm{i}}\right)= \\
& =S_{2}\left(\lambda_{\mathrm{i}}, \lambda^{\prime}\right)\left[\alpha_{\mathbf{A}}\left(\lambda_{\mathrm{i}}\right) \alpha_{\mathbf{B}}\left(\lambda_{\mathrm{i}}\right)+\alpha_{\mathbf{A}}\left(\lambda_{\mathrm{i}}\right) \alpha_{\mathbf{B}}\left(\lambda_{\mathrm{i}}\right)\right] .
\end{aligned}
$$

Comparison between (3.9) and (3.10) shows that the transition amplitude $\mathcal{A}\left(\lambda, \lambda^{\prime}\right)$ has the same expression for spontaneous and stimulated processes. The enhancement due to stimulated emission (multiplication by $\sqrt{2}$ for the stimulated amplitudes) is balanced by other effects associated with the indiscernability of the input and emitted photons.

This means that the two level atom of figure 3 behaves not at all as an "amplifier" but rather as a noise source independent of the input photon. The process in which a "cloned " photon is emitted in the input mode $\lambda_{i}$ has just the same contribution as any process where a "noise" photon is emitted in an empty mode $\lambda$. It must be emphasized that this remark is valid only when the two photons (input photon and photon emitted by the "amplifier") are detected, i.e. when a correlation signal is studied. If the intensity of the field was measured behind the "amplifier" (only one photon detected), evidence for amplification could be obtained (the output intensity would be greater than the sum of the input and noise intensities).

\section{Diagrammatic representation of the transition amplitudes.}

We want to give now a diagrammatic representation of the transition amplitudes which will be useful for discussing the role of stimulated and spontaneous processes in the low intensity interference experiment of figure 2.

We associate the diagrams respectively sketched on figure 4 and figure 5 to the stimulated and spontaneous amplitudes. Note that transmission or reflection by the beam splitter are now included explicitly in the transition amplitudes. We will have therefore to discuss in more details in this section the transmission and reflection amplitudes.

As previously, the enhancement due to stimulated emission (multiplication by $\sqrt{2}$ of the emission ampli- 
tude) is not the only difference between the diagrams of figures 4 and 5 . As a matter of fact, the two detected photons are identical for stimulated processes (Fig. 4) but distinct for spontaneous ones (Fig. 5). There is therefore one possibility for detecting them at the two detectors in the first case (Fig. 4) but two in the second one (Fig. 5). Furthermore the amplitude describing the reflection of one photon and the transmission of the other one by the beam splitter has not the same value in the two cases.

When the two incident photons are distinct $\left(\lambda \neq \lambda_{\mathrm{i}}\right.$; see Fig. 5), such an amplitude is simply equal to $t r$ (for each diagram of figure 5) where $t$ and $r$ are the one photon transmission and reflection amplitudes.

When the two incident photons are identical (Fig. 4), the correct evaluation of this amplitude requires to symmetrize the pre and postcollision states. Let us note :

$$
\left|\Phi_{\mathrm{II}}\right\rangle=\left|1 \lambda_{\mathrm{l}}, 2 \lambda_{\mathrm{I}}\right\rangle
$$

the precollision state (photons 1 and 2 both in the incident mode denoted here $\lambda_{\mathrm{I}}$ ) and :

$$
\left|\Phi_{\mathrm{RT}}\right\rangle=\frac{1}{\sqrt{2}}\left(\left|1 \lambda_{\mathrm{T}}, 2 \lambda_{\mathrm{R}}\right\rangle+\left|1 \lambda_{\mathrm{R}}, 2 \lambda_{\mathrm{T}}\right\rangle\right)
$$

the postcollision one (one photon in the transmitted

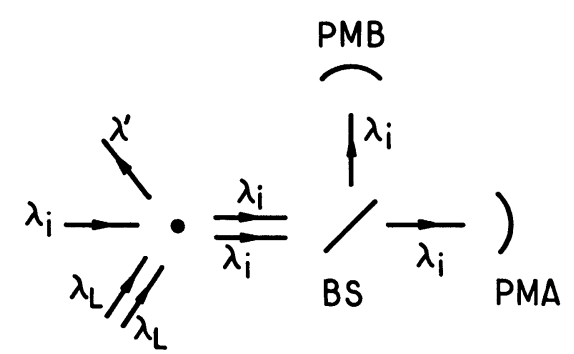

Fig. 4. - Diagrammatic representation of the stimulated amplitude $\mathcal{A}\left(\lambda_{\mathrm{i}}, \lambda^{\prime}\right)$ (experiment of fig. 1). The atom emits a photon $\lambda_{i}$ (emission stimulated by the presence of the input photon) and a photon $\lambda^{\prime}$ (spontaneous emission). One of the two $\lambda_{\mathrm{i}}$ photons is transmitted through BS and detected at PMA, the other is reflected on BS and detected at PMB. mode $\lambda_{T}$ and the other in the reflected one $\lambda_{R}$ ) : $\left|\Phi_{\mathrm{II}}\right\rangle$ is of course symmetrical and $\left|\Phi_{\mathrm{RT}}\right\rangle$ has been symmetrized and normalized. One then gets the transmission-reflection amplitude for two identical photons :

$$
\left\langle\Phi_{\mathrm{RT}}\left|S_{\mathrm{BS}}\right| \Phi_{\mathrm{II}}\right\rangle=\frac{1}{\sqrt{2}}(r t+r t)=r t \sqrt{2}
$$

where $S_{\mathrm{BS}}$ is the $S$ matrix corresponding to the action of the beam splitter.

As a consequence, one concludes again that the enhancement due to stimulated emission is balanced by effects associated with the indistinguishability of the two photons emerging from the amplifier (emission amplitude is multiplied by $\sqrt{2}$, transmission-reflection amplitude is multiplied by $\sqrt{2}$ but there is only one diagram for the stimulated process of figure 4 whereas there are two for the spontaneous one of figure 5).

Remarks : i) The enhancement by a factor $\sqrt{2}$ which appeared in $\S 3$ in the detection amplitude (see eq. (3.8) is no longer present since the two photons are now detected in different modes. In fact, it has been replaced by the factor $\sqrt{2}$ in the transmissionreflection amplitude.

ii) For a 50-50 beam splitter $(r=t=1 / \sqrt{2})$, the probability that one photon is transmitted and the other reflected is obviously $1 / 2$ when the two incident photons are distinct. Reading too rapidly the Feynman's lecture on "Identical particules » [36] may lead to the wrong idea that this probability is $1 / 3$ when the two incident photons are identical [19-22]. The previous discussion shows on the contrary that this probability :

$$
\left|\left\langle\Phi_{\mathrm{RT}}\left|S_{\mathrm{BS}}\right| \Phi_{\mathrm{II}}\right\rangle\right|^{2}=2 r^{2} t^{2}
$$

has exactly the same value as for non identical photons $(1 / 2$ for $r=t)$.

\section{Delay dependence of the correlation signal.}

We have demonstrated in the previous sections that the correlation signal corresponds to the detection of photons emitted by two independent sources, the input source and the " amplifier » behaving as a noise
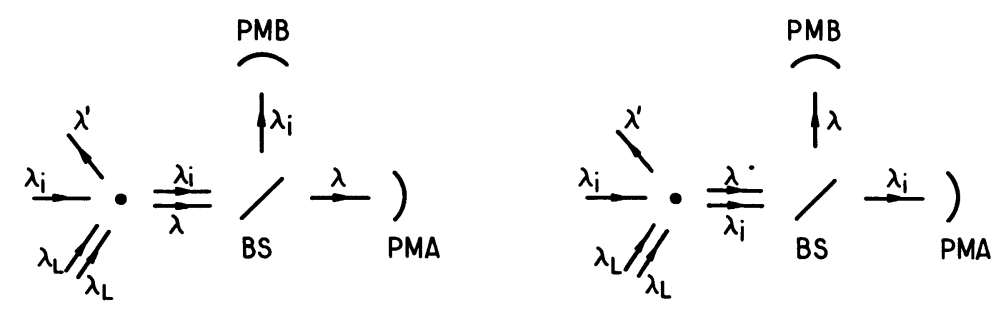

Fig. 5. - The two diagrams associated with the spontaneous amplitude $\mathcal{A}\left(\lambda, \lambda^{\prime}\right)\left(\lambda \neq \lambda_{\mathrm{i}}\right)$. The atom spontaneously emits two photons $\lambda$ and $\lambda^{\prime}$. One of the two photons $\lambda_{\mathrm{i}}$ and $\lambda$ coming on BS is transmitted and detected at PMA, the other is reflected and detected at PMB. 
source. Actually, the correlation signal $P$ is given by (from Eqs. (2.11), (3.9) and (3.10)) :

$$
\begin{aligned}
P=C R T \sum_{\lambda^{\prime}} \mid \sum_{\lambda} S_{2}\left(\lambda, \lambda^{\prime}\right)\left(\alpha_{\mathbf{A}}\left(\lambda_{\mathrm{i}}\right) \alpha_{\mathbf{B}}(\lambda)+\right. & \\
& \left.+\alpha_{\mathbf{A}}(\lambda) \alpha_{\mathbf{B}}\left(\lambda_{\mathrm{i}}\right)\right)\left.\right|^{2}
\end{aligned}
$$

(with $\omega_{\lambda} \simeq 2 \omega_{\mathrm{L}}-\omega_{0} ; \omega_{\lambda^{\prime}} \simeq \omega_{0}$ ) where the input mode $\lambda_{\mathrm{i}}$ is included in the sum over $\lambda$. It can thus be written :

$$
P=C \overline{\left|\mathcal{E}_{\mathrm{A}}^{(\mathrm{i})} \boldsymbol{E}_{\mathrm{B}}^{(\mathrm{n})}+\boldsymbol{E}_{\mathrm{A}}^{(\mathrm{n})} \boldsymbol{E}_{\mathrm{B}}^{(\mathrm{i})}\right|^{2}}
$$

where $\boldsymbol{\varepsilon}_{A}^{(\mathbf{i})}$ and $\boldsymbol{\varepsilon}_{A}^{(\mathbf{n})}$ appear as classical fields which would be emitted by the input and noise sources :

$$
\begin{aligned}
& \boldsymbol{E}_{\mathbf{A}^{(i)}}^{(\mathbf{i})}=t \alpha_{\mathbf{A}}\left(\lambda_{\mathrm{i}}\right) \\
& \boldsymbol{\varepsilon}_{\mathrm{A}^{(\mathrm{n})}}=t \sum_{\lambda} S_{2}\left(\lambda, \lambda^{\prime}\right) \alpha_{\mathrm{A}}(\lambda)
\end{aligned}
$$

(same definitions for $\boldsymbol{E}_{\mathbf{B}}^{(\mathbf{i})}$ and $\boldsymbol{E}_{\mathbf{B}}^{(\mathbf{n})}$ with $r$ replacing $t$ ); the summation over $\lambda^{\prime}$ in (5.1) is considered in (5.2) as the average over the various realizations of the noise field. The expression (5.2) makes a clear connection with the Hanbury-Brown and Twiss effect [1418] which appears when intensity correlations between two independent classical sources are studied. Although stimulated emission is compensated (see $\S 3$ ), photon correlations will be observed at the output of the "amplifier". We calculate now these correlations, i.e. we calculate the delay dependence of $P$.

We have first to perform the angular integration of equation (5.1) (summation over shells of modes $\lambda$ of frequency $\omega_{\lambda}$ ). As usually, retardation effects appear at this stage : the detection times $t_{\mathrm{A}}$ and $t_{\mathrm{B}}$ are replaced by :

$$
\tau_{\mathrm{A}}=t_{\mathrm{A}}-r_{\mathrm{A}} / c ; \quad \tau_{\mathrm{B}}=t_{\mathrm{B}}-r_{\mathrm{B}} / c
$$

( $r=0$ corresponds to the "amplifier" position). More precisely, the angular integration gives :

$$
\begin{aligned}
P=C^{\prime} R T & \sum_{\lambda^{\prime}} \mid \int \mathrm{d} \omega_{\lambda} g\left(\omega_{\lambda}\right) s_{2}\left(\omega_{\lambda}, \omega_{\lambda^{\prime}}\right) \times \\
& \left.\left(\mathrm{e}^{-i \omega_{\lambda} \tau_{\mathbf{A}}} \mathrm{e}^{-i \omega_{\mathrm{i}} \tau_{\mathbf{B}}}+\mathrm{e}^{-i \omega_{\lambda} \tau_{\mathrm{B}}} \mathrm{e}^{-i \omega_{\mathbf{i}} \tau_{\mathbf{A}}}\right)\right|^{2}
\end{aligned}
$$

where $s_{2}\left(\omega_{\lambda}, \omega_{\lambda^{\prime}}\right)$ contains the frequency dependence of $S_{2}\left(\lambda, \lambda^{\prime}\right)\left(C^{\prime}\right.$ is a new multiplicative constant). The variation of $s_{2}$ is discussed in details elsewhere (see in particular Eq. (5.10) of [33]). From this discussion, one can write :

$$
\begin{aligned}
& g\left(\omega_{\lambda}\right) s_{2}\left(\omega_{\lambda}, \omega_{\lambda^{\prime}}\right)= \\
& \quad=\delta\left(\omega_{\lambda}+\omega_{\lambda^{\prime}}-2 \omega_{\mathrm{L}}\right)\left(1 /\left(\omega_{\lambda^{\prime}}-\omega_{0}+\frac{i \Gamma}{2}\right)\right) .
\end{aligned}
$$

The $\delta$ function expresses the energy conservation in the multiphoton process of figure 3 . The second factor describes the resonant behaviour of this process for $\omega_{\lambda} \simeq 2 \omega_{\mathrm{L}}-\omega_{0}$ (i.e. $\omega_{\lambda^{\prime}} \simeq \omega_{0}$ ) within a linewidth $\Gamma$. The two other resonances which appear in $s_{2}$ (for $\omega_{\lambda}=\omega_{\mathrm{L}}$ and $\omega_{\lambda} \simeq \omega_{0}$ ) are suppressed by the filtering amplitude $g\left(\omega_{\lambda}\right)$ (see Eq. (2.5)).

Now the frequency integration of (5.5) (summation over $\omega_{\lambda}$ ) is straightforward because of the presence of the $\delta$ function :

$$
\begin{aligned}
P=C^{\prime} & R T \sum_{\lambda^{\prime}}\left(1 /\left(\left(\omega_{\lambda^{\prime}}-\omega_{0}\right)^{2}+\frac{\Gamma^{2}}{4}\right)\right) \times \\
\times & \left|\mathrm{e}^{-i \omega_{\lambda} \tau_{\mathbf{A}}} \mathrm{e}^{-i \omega_{\mathrm{i}} \tau_{\mathbf{B}}}+\mathrm{e}^{-i \omega_{\lambda} \tau_{\mathbf{B}}} \mathrm{e}^{-i \omega_{\mathbf{i}_{1} \tau_{\mathbf{A}}}}\right|^{2}
\end{aligned}
$$

with $\omega_{\lambda}=2 \omega_{\mathbf{L}}-\omega_{\lambda^{\prime}}$. Since $\omega_{\mathrm{i}}=2 \omega_{\mathbf{L}}-\omega_{0},(5.7)$ becomes :

$$
\begin{aligned}
P=C^{\prime} R T \sum_{\lambda^{\prime}}\left(1 /\left(\left(\omega_{\lambda^{\prime}}\right.\right.\right. & \left.\left.\left.-\omega_{0}\right)^{2}+\frac{\Gamma^{2}}{4}\right)\right) \times \\
& \times\left|1+\mathrm{e}^{i\left(\omega_{\lambda^{\prime}}-\omega_{0}\right) \tau}\right|^{2}
\end{aligned}
$$

where $\tau$ is the delay between the two detections :

$$
\tau=\tau_{\mathbf{B}}-\tau_{\mathbf{A}}
$$

( $P$ depends only on $\tau$ because of the stationarity of the sources). The summation over $\lambda^{\prime}$ finally gives :

$$
P=C^{\prime \prime} R T\left(1+\mathrm{e}^{-\Gamma|\tau| / 2}\right) .
$$

One recognizes the characteristic delay dependence of the Hanbury-Brown and Twiss effect due to interference between the two paths of figure 5 where the two independent sources have respectively a zero width (for the "input » source) and a linewidth $\Gamma$ (for the " amplifier »).

\section{Conditions for predominance of « one input pho- ton » contribution to the signal.}

Up to now, we have only discussed the " one input photon" contribution to the signal where the two detectors are excited by an input photon and a noise one. We have thus neglected the " two input photons " contribution where two input photons are detected as well as the "no input photon " contribution where two noise photons are detected. We want in this section to find what conditions are required for the " one input photon" contribution to be predominant. We have thus to evaluate, at least qualitatively, these three contributions.

The " one input photon" contribution, studied in the previous section, can be written :

$$
P^{(\text {in })}=2 R T I^{(\mathrm{i})} I^{(\mathrm{n})}(1+\exp (-\Gamma|\tau| / 2))
$$


where $I^{(\mathbf{i})}, I^{(\mathrm{n})}$ are the input and noise intensities which would be measured by a detector at the output of the amplifier (the multiplicative factor appearing in equation (6.1) is calculated by taking $\tau$ infinite). The «two input photon" contribution $\mathrm{P}^{(\mathrm{ii})}$ and the " no input photon " contribution $P^{(\mathrm{nn})}$ may be expressed as :

$$
\begin{aligned}
P^{(\mathrm{ii})} & =R T I^{(\mathrm{i})^{\bullet}} I^{(\mathrm{i})} g^{(\mathrm{ii})}(\tau) \\
P^{(\mathrm{nn})} & =R T I^{(\mathrm{n})} I^{(\mathrm{n})} g^{(\mathrm{nn})}(\tau)
\end{aligned}
$$

where $g^{(\mathrm{ii})}$ and $g^{(\mathrm{nn})}$ are the normalized intensity correlation functions associated respectively with the input and noise sources. Two conditions are needed for the " one input photon " contribution to be predominant :

$$
\begin{aligned}
& P^{(\mathrm{ii})} \ll P^{(\mathrm{in})} \\
& P^{(\mathrm{nn})} \ll P^{(\mathrm{in})} .
\end{aligned}
$$

We want to emphasize that these two conditions cannot be both satisfied if the input and noise sources can be considered as classical. In such a case, it is known [37-42] that :

$$
\begin{aligned}
& g^{(\mathrm{ii})}(0) \geqslant 1 \\
& g^{(\mathrm{nn})}(0) \geqslant 1
\end{aligned}
$$

which implies (see Eqs. (6.1), (6.2)) :

$$
P^{(\mathrm{ii})} P^{(\mathrm{nn})} \geqslant\left(P^{(\mathrm{in})} / 4\right)^{2} \text {. }
$$

This is clearly not compatible with the two conditions (6.3)

Either the amplifier or the input source must therefore be non classical if one wants to study the " one input photon" contribution. The amplifier is a non classical source if it involves only one atom (antibunching effect [43-49]). But if the amplifier contains many atoms, it can be considered as classical. The character of the input source has thus a great importance. Let us emphasize that attenuating the input source is not sufficient when it is classical [50]. The " two input photon" contribution can indeed be reduced (first condition (6.3) satisfied) but the " no input photon" contribution thus becomes predominant (second condition (6.3) not satisfied).

Remark : Constructing "one photon source » is now achievable. Radiative cascades indeed provide couples of correlated photons [51-52] (see also [53] and [32]). One of them can be used as the input photon and the other for opening observation gates [54]. The input source thus behaves as a non classical " one photon source» [54-55]. If such a device was used, the experiment sketched on figure 1 would become a three events correlations set-up : detection of one photon emitted in the radiative cascade (the " trigger » photon), detection of the second one (the "input " photon), detection of the photon emitted by the amplifier (the noise photon).

\section{Application to correlation signals in interference experiments with « one photon amplifier ».}

Now we come to the set-up of figure 2 where the " amplifier " of figure 3 is put in one arm (denoted 1) of a Mach-Zender interferometer [22]. We have to study the correlation signal $P$ delivered by the two detectors A (located behind the beam splitter BS1) and $B$ (located at one output of the interferometer). The quantitative calculation of $P$ can be done in the same manner as in former sections (which correspond to the correlation signal of figure 1 ). We do not detail this calculation but rather give the results and discuss their interpretation.

The correlation signal can be written in terms of the transition amplitudes represented by the diagrams of figures 6, 7 and 8. The transition amplitudes of figure 6 correspond to the paths where the input photon is transmitted through the beam splitter BS0 and stimulates the emission by the atom of a "cloned photon ", the two identical photons $\lambda_{i}$ being then destroyed in the two detectors $A$ and $B$. The notation $\mathcal{A}_{1}\left(\lambda_{\mathrm{i}}, \lambda^{\prime}\right)$ means first that the incident photon enters the arm 1 of the interferometer and second that the " amplifier " emits two photons in the modes $\lambda_{i}$ and $\lambda^{\prime}$. For the amplitudes $\mathcal{A}_{1}\left(\lambda, \lambda^{\prime}\right)$ represented on figure 7 , the input photon is still transmitted through the beam splitter BS0 and destroyed by one of the detectors, but the photon destroyed by the other detector is now spontaneously emitted by the "amplifier" in a mode $\lambda$ different from $\lambda_{i}$ (《 noise » photon). As in figure 5 , two possibilities contribute to these amplitudes corresponding to the detection of $\lambda_{i}$ on $A$ and $\lambda$ on $\mathrm{B}$ or vice versa. Finally, the amplitudes $\mathcal{A}_{2}\left(\lambda, \lambda^{\prime}\right)$ (Fig. 8) correspond to processes where the input photon is reflected by the beam splitter BS0, passes through the arm 2 of the interferometer and is destroyed on the detector B. The photon which

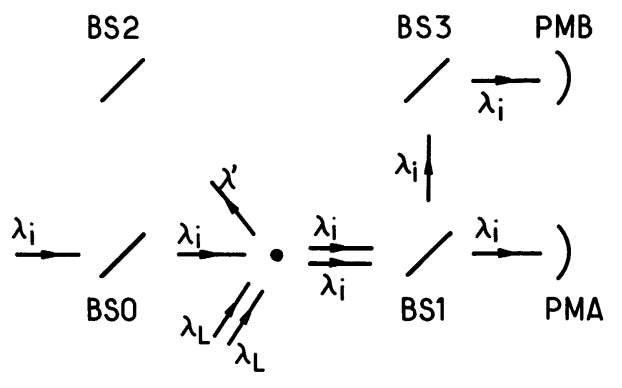

Fig. 6. - Diagrammatic representation of the stimulated amplitude $\mathcal{A}_{1}\left(\lambda_{\mathrm{i}}, \lambda^{\prime}\right)$. The input photon $\lambda_{\mathrm{i}}$ is transmitted through BS0. The atom emits of $\lambda_{i}$ photon (stimulated emission). One of the two $\lambda_{\mathrm{i}}$ photons is transmitted through BS1 and detected at PMA. The other is reflected on BS1. reflected on BS3 and detected at PMB. 


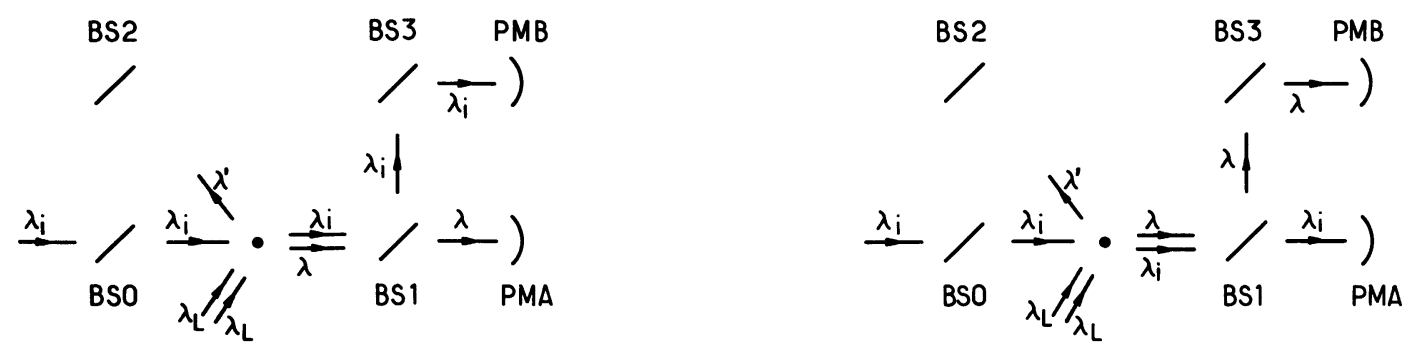

Fig. 7. - The two diagrams associated with the spontaneous amplitude $\mathcal{A}_{1}\left(\lambda, \lambda^{\prime}\right)\left(\lambda \neq \lambda_{\mathrm{i}}\right)$. The input photon $\lambda_{\mathrm{i}}$ is transmitted through BS0. The atom spontaneously emits a $\lambda$ photon. One of the two photons $\lambda$ and $\lambda_{\mathrm{i}}$ coming on BS1 is transmitted and detected at PMA. The other is reflected on BS1, reflected on BS3 and detected at PMB.

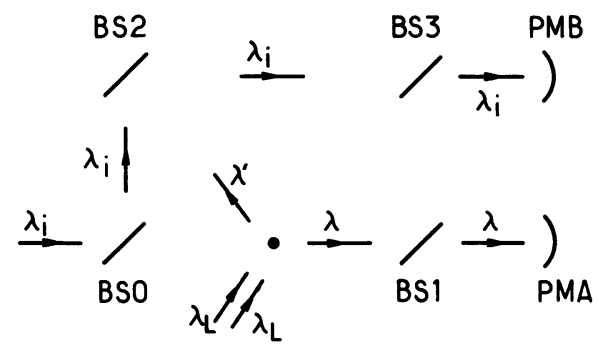

Fig. 8. - Diagrammatic representation of the spontaneous amplitude $\mathcal{A}_{2}\left(\lambda, \lambda^{\prime}\right)$. The input photon $\lambda_{i}$ is reflected on BS0, reflected on BS2, transmitted through BS3 and detected at PM3. The atom spontaneously emits a $\lambda$ photon which is transmitted through BS1 and detected at PMA.

excites the detector $\mathrm{A}$ is then necessarily a noise photon emitted by the amplifier. All these amplitudes (Figs. 6, 7 and 8) describe indistinguishable paths from the initial state to a given final state when the value of $\lambda^{\prime}$ is fixed. Consequently, they interfere. On the other hand, the paths corresponding to different final states (different values of $\lambda^{\prime}$ ) do not interfere. The expression of $\mathbf{P}$ in terms of the transition amplitudes is thus :

$$
P=C \sum_{\lambda^{\prime}}\left|\sum_{\lambda}\left(\mathcal{A}_{1}\left(\lambda, \lambda^{\prime}\right)+\mathcal{A}_{2}\left(\lambda, \lambda^{\prime}\right)\right)\right|^{2}
$$

( $C$ is a multiplicative constant).

Stimulated emission by the "amplifier" only appears in $\mathcal{A}_{1}\left(\lambda_{\mathrm{i}}, \lambda^{\prime}\right)$ while spontaneous emission processes occur for all other amplitudes. But, in the same manner as previously, the enhancement due to stimulated emission (multiplication by $\sqrt{2}$ of the emission amplitude) is balanced by effects associated with the indistinguishability of the two photons emerging from the amplifier (see $\S 4$ ). So the input mode has not to be distinguished from the others in the summation over $\lambda$ of equation (7.1), which means that the two level atom of fig. 3 behaves not as an " amplifier" but rather as an independent noise source (when a correlation signal is studied : see $\S 3$ ).

One can now make explicit the transition amplitudes (as in $\S 3$ ) and perform the summation over $\lambda$ in equation (7.1) (as was done in $\S 5$ ). One then obtains an expression of $P$ similar to the expression (5.8) :

$$
\begin{aligned}
P=C^{\prime} \sum_{\lambda^{\prime}} & \left(1 /\left(\left(\omega_{\lambda^{\prime}}-\omega_{0}\right)^{2}+\frac{\Gamma^{2}}{4}\right)\right) \times \\
& \left|r_{1} t_{1}\left(1+\mathrm{e}^{i\left(\omega_{\lambda^{\prime}}-\omega_{0}\right) \tau}\right)+r_{2} t_{1} \mathrm{e}^{i \Phi}\right|^{2}
\end{aligned}
$$

where $r_{1}$ and $t_{1}$ are the reflexion and transmission amplitudes of the beam splitter BS1, $r_{2}$ the reflexion amplitude of BS2 (BS0 and BS3 are supposed to be 50-50 beam-splitter; the corresponding factors $1 / 2$ are included in $C^{\prime}$ ); $\Phi$ is the phase difference between the two arms of the interferometer. The two terms containing respectively $r_{1}$ and $r_{2}$ correspond to the amplitudes $\mathcal{A}_{1}$ (Fig. 7) and $\mathcal{A}_{2}$ (Fig. 8). The first term is itself a sum of two amplitudes corresponding to the two diagrams of figure 7 (same difference between them as in Eq. (5.8)). The relation between this first term and the second one can be intuitively understood. As a matter of fact, the diagram of figure 8 and the first diagram of figure 7 only differ by the distance covered by the input photon from the beam splitter BS0 to the detector B. So the amplitude $\mathcal{A}_{2}$ is related to the first part of the amplitude $\mathcal{A}_{1}$ through the phase factor $\mathrm{e}^{i \Phi}$.

Consider first the case of the zero delay $(\tau=0$ in Eq. (7.2)) : $P$ varies as :

$$
P(0)=C^{\prime \prime} t_{1}^{2}\left|2 r_{1}+r_{2} \mathrm{e}^{i \Phi}\right|^{2} .
$$

This must be compared to the intensity $I_{B}$ which would be measured by the detector B in the absence of the amplifier :

$$
I_{\mathrm{B}} \simeq\left|r_{1}+r_{2} \mathrm{e}^{i \Phi}\right|^{2} .
$$

The difference between these two expressions can be attributed to the Hanbury-Brown and Twiss effect discussed previously : the amplitude associated with the arm 1 is multiplied by a factor 2 because of the constructive interference between the two diagrams of figure 7 .

When the delay is larger than the coherence time of the noise source (i.e. when $\tau \gg \Gamma^{-1}$ ), the HanburyBrown and Twiss effect disappears (i.e. there is no longer interference between the two diagrams of 
figure 7). The expression (7.2) of $P$ reduces to :

$$
P(\infty)=C^{\prime \prime} t_{1}^{2}\left(\left|r_{1}+r_{2} e^{i \Phi}\right|^{2}+r_{1}^{2}\right) .
$$

The first term corresponds to the detection of the input photon by B (same behaviour as the intensity in equation (7.4)) whereas the second term corresponds to the detection of a noise photon by $B$ and of the input one by $A$.

In the intermediate case, the expression of $P$ can be calculated by integrating over $\omega_{\lambda^{\prime}}$ in equation (7.2). One gets :

$$
\begin{aligned}
& P(\tau)=C^{\prime \prime} t_{1}^{2} \times \\
& \quad \times\left[r_{2}^{2}+2 r_{1}\left(r_{1}+r_{2} \cos \Phi\right)(1+\exp (-\Gamma|\tau| / 2))\right]
\end{aligned}
$$

(exponential decrease with a time constant $2 \Gamma^{-1}$ from $P(0)$ (Eq. 7.3) to $P(\infty)$ (Eq. 7.5)).

\section{Considerations about the fringe contrast.}

The variation of the correlation signal $P$ versus the phase difference $\Phi$ can be characterized by a fringe contrast $\gamma$. We want to show in this section that $\gamma$ obeys an inequality which has a very intuitive meaning.

From equation (7.1), $P$ can be split into three terms :

$$
P=P_{1}+P_{2}+P_{\text {int }}
$$

with :

$$
\begin{aligned}
P_{1} & =C \sum_{\lambda^{\prime}}\left|\sum_{\lambda} \mathcal{A}_{1}\left(\lambda, \lambda^{\prime}\right)\right|^{2} \\
P_{2} & =C \sum_{\lambda^{\prime}}\left|\sum_{\lambda} \mathcal{A}_{2}\left(\lambda, \lambda^{\prime}\right)\right|^{2} \\
P_{\text {int }} & =C \sum_{\lambda^{\prime}}\left(\sum_{\lambda} \mathcal{A}_{1}\left(\lambda, \lambda^{\prime}\right)\right)^{*}\left(\sum_{\lambda} \mathcal{A}_{2}\left(\lambda, \lambda^{\prime}\right)\right)+\text { c.c. }
\end{aligned}
$$

The two first terms, $P_{1}$ and $P_{2}$, do not depend on the phase difference. The fringe contrast $\gamma$ can thus be written :

$$
\gamma=P_{\text {int, max }} /\left(P_{1}+P_{2}\right)
$$

where $P_{\text {int, } \max }$ is the maximum value taken by $P_{\text {int }}$ when $\Phi$ varies. But $P_{\text {int }}$ obeys a Schwarz type inequality :

$$
P_{\text {int }} \leqslant 2 \sqrt{P_{1} P_{2}}
$$

which leads to the following inequality for the contrast :

$$
\gamma \leqslant 2 \sqrt{P_{1} P_{2}} /\left(P_{1}+P_{2}\right) .
$$

In order to discuss the meaning of this inequality, let us consider the experiments sketched on figure 9 . The output beam splitter BS3 and the detector B are replaced by two detectors B1 and B2 located respectively in arms 1 and 2 of the interferometer. The correlation signal measured by the detectors $A$ and B1 (resp. A and B2) is just the signal $P_{1}$ (resp. $P_{2}$ ) defined by equation (8.2). Actually, the transition amplitudes from the initial state to final states where A and B1 (resp. B2) are excited are the amplitudes $\mathcal{A}_{1}\left(\lambda, \lambda^{\prime}\right)$ (resp. $\left.\mathcal{A}_{2}\right)$ (see Figs. 7 and 8).

Let us now define the two quantities :

$$
\pi_{1}=P_{1} /\left(P_{1}+P_{2}\right) ; \quad \pi_{2}=P_{2} /\left(P_{1}+P_{2}\right)
$$

From the previous discussion, $\pi_{1}$ (resp. $\pi_{2}$ ) can be considered as the conditional probability for the detector B1 (resp. B2) to be excited knowing that either $\mathrm{A}$ and $\mathrm{B} 1$ or $\mathrm{A}$ and $\mathrm{B} 2$ are excited. The inequality (8.5) can thus be written :

$$
\gamma \leqslant 2 \sqrt{\pi_{1} \pi_{2}} .
$$

This inequality has been derived here from the expression (7.1) of the correlation signal which is valid for the amplifier of figure 3. But it can be deduced directly from the expression of the signals in terms of the electric fields $E_{\mathrm{A}}, E_{\mathrm{B}}, E_{\mathrm{B} 1}, E_{\mathrm{B} 2}$ experienced by the detectors A and B (Fig. 2). B1 and B2 (Fig. 9). The correlation signals can indeed be written in the Heisenberg point of view as :

$$
\begin{aligned}
& P=\left\langle E_{\mathrm{A}}^{-} E_{\mathrm{B}}^{-} E_{\mathrm{B}}^{+} E_{\mathrm{A}}^{+}\right\rangle \\
& P_{1}=\left\langle E_{\mathrm{A}}^{-} E_{\mathrm{B} 1}^{-} E_{\mathrm{B} 1}^{+} E_{\mathrm{A}}^{+}\right\rangle
\end{aligned}
$$

(same Eq. for $P_{2}$ ). Using :

$$
E_{\mathrm{B}}=\left(E_{\mathrm{B} 1}+E_{\mathrm{B} 2}\right) / \sqrt{2}
$$

one obtains :

$$
P=\left(P_{1}+P_{2}+P_{\text {int }}\right) / 2
$$

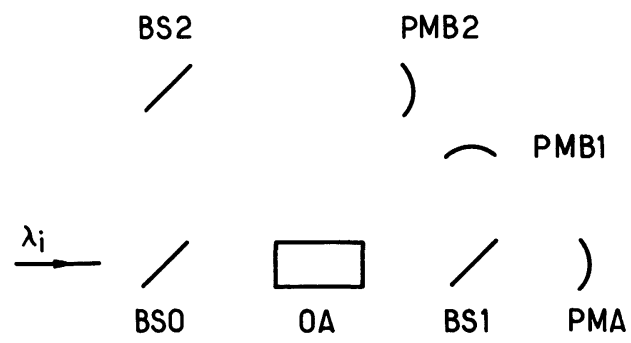

Fig. 9. - Experiment complementary to the experiment of figure 2. Correlations are studied using the detector PMA located behind BS1 and either PMB1 located in the arm 1 or PMB2 located in the arm 2. 
with :

$$
P_{\mathrm{int}}=\left\langle E_{\mathrm{A}}^{-}\left(E_{\mathrm{B} 1}^{-} E_{\mathrm{B} 2}^{+}+E_{\mathrm{B} 2}^{-} E_{\mathrm{B} 1}^{+}\right) E_{\mathrm{A}}^{+}\right\rangle .
$$

Now, the interference term obeys a Schwarz type inequality which is just the inequality (8.4). The inequalities (8.5) and (8.7) bearing on the fringe contrast are therefore valid whatever the design of the amplifier of figure 2 may be.

If the amplifier was an ideal duplicator, $\pi_{2}$ would be equal to zero, meaning that it is impossible to detect a photon on A and a photon on B2. In these conditions, the inequality (8.7) would imply the absence of interference effect $(\gamma=0)$. But as soon as noise photons can be emitted by the amplifier, $\pi_{2}$ is no longer zero (one can detect a noise photon on $A$ and the input photon on B2). So, quantum mechanics does allow the presence of interference (equation (8.7) does not imply $\gamma=0$ ).

Remark : For the amplifier sketched on figure 3, $\gamma, \pi_{1}$ and $\pi_{2}$ can be evaluated from equation (7.6). One finds :

$$
\gamma / \sqrt{\pi_{1} \pi_{2}}=\sqrt{2(1+\exp (-\Gamma|\tau| / 2))} .
$$

The inequality (8.7) is of course always satisfied. The equality between the two members of $(8.7)$ is reached only for $\tau=0$.

\section{Conclusion.}

We have shown that when correlations in single photon amplification are studied (Fig. 1), the results can be analysed without referring to stimulated emission. More precisely, the enhancement due to stimulated emission is exactly compensated by effects associated with the indistinguishability of the two photons going out the amplifier. The atom of figure 3 thus appears not at all as an amplifier but rather as a noise source independent of the input photon. Obviously, this would no longer be true if an intensity signal was measured or if several photons were coming on the amplifier : amplification by stimulated emission does exist.

The result obtained in this paper seems to be in agreement with the experimental observations of Scarl and Smith [4] and Blake and Scarl [8]. But their experimental conditions were different from those supposed in this paper : their input source is a classical one and cannot be considered as a one photon source : their optical amplifier is a light gain tube different from ours. So further analysis is required for understanding completely these experimental observations.

When the amplifier is put in one arm of a MachZender interferometer, it behaves again as a noise source and not at all as an ideal photon duplicator. In particular, it cannot be expected to discriminate stimulated effects from spontaneous ones by some spatial selection. As a consequence, quantum mechanics predicts that the correlation signal registered by detectors $A$ and $B$ is sensitive to the phase difference between the two arms of the interferometer [23].

\section{Acknowledgments.}

We are grateful to C. Cohen-Tannoudji and J. Dalibard for constant help and encouragement. We want also to thank A. Aspect, B. Cagnac and A. Gozzini for stimulating discussions.

\section{References}

[1] Gordon, J. P., Walker, L. R. and Louisell, W. H., Phys. Rev. 130 (1963) 806.

[2] Loudon, R., Phys. Rev. A 2 (1970) 267.

[3] Carusotto, S., Phys. Rev. A 11 (1975) 1629.

[4] SCarl, D. B. and Smith, S. R., Phys. Rev. A 10 (1974) 709 and $A 14$ (1976) 2355.

[5] Mandel, L., Phys. Rev. A 14 (1976) 2351.

[6] Abraham, N. B. and Smith, S. R., Phys. Rev. A 15 (1977) 421.

[7] Rockower, E. B., Abraham, N. B. and Smith, S. R., Phys. Rev. A 17 (1978) 1100.

[8] Blake, G. D. and Scarl, D., Phys. Rev. A 19 (1979) 1948.

[9] MCNeIl, K. J. and Walls, D. F., J. Phys. A 8 (1975) 104.

[10] Mohr, U., Optics Commun. 41 (1982) 21.

[11] Bendjaballah, C. and Oliver, G., Phys. Rev. A 28 (1983) 294.
[12] Wootters, W. K. and Zurek, W. H., Nature 299 (1982) 802.

[13] Milonni, P. W. and Hardies, M. L., Phys. Lett. 92A (1982) 321.

[14] Hanbury Brown, R. and Twiss, R. Q., Nature 177 (1956) 27 ; 178 (1956) 1046 ; Proc. R. Soc. London; 242A (1956) 300 ; 243A (1957) 291.

[15] Twiss, R. Q., Little, A. G. and Hanbury Brown, R., Nature 180 (1957) 324.

[16] Fano, U., Am. J. Phys. 29 (1961) 539.

[17] Mandel, L. and Wolf, E., Rev. Mod. Phys. 37 (1965) 231.

[18] Cohen-Tannoudji, C., Proc. of Les Houches Summer School, July 1982, eds Grynberg G. and Stora R. (North Holland, in the press).

[19] Garuccio, A., Rapisarda, V. and Vigier, J. P., Phys. Lett. 90A (1982) 17.

[20] Selleri, F., Foundations Phys. 12 (1982) 1087. 
[21] Andrade e Silva, J., Selleri, F. and Vigier, J. P., Lett. Nuovo Cim. 36 (1983) 503.

[22] GozzinI, A., Proc. Symp. on Wave-Particle Dualism, eds Diner S., Fargue D., Lochak G. and Selleri F. (Reidel, in the press).

[23] Loudon, R., Optics Commun. 45 (1983) 361.

[24] Friberg, S. and Mandel, L., Optics Commun. 46 (1983) 141.

[25] Mollow, B. R., Phys. Rev. A 5 (1972) 2217.

[26] Wu, F. Y., Ezekiel, S., Ducloy, M. and Mollow, B. R., Phys. Rev. Lett. 38 (1977) 1077.

[27] Cohen-Tannoudj, C. and Reynaud, S., in Multiphoton Processes, eds Eberly J. H. and Lambropoulos P. (Wiley) 1978, p. 103.

[28] Cohen-Tannoudi, C. and Reynaud, S., J. Phys. B 10 (1977) 345.

[29] Reynaud, S., Ann. Phys. Fr. 8 (1983) 315.

[30] Cagnac, B., Grynberg, G., Biraben, F., J. Physique 34 (1973) 845.

[31] Cagnac, B., Grynberg, G., Biraben, F. and Bassini, M., Phys. Rev. Lett. 37 (1976) 283.

[32] Aspect, A., Roger, G., Reynaud, S., Dalibard, J. and Cohen-Tannoudj, C., Phys. Rev. Lett. 45 (1980) 617.

[33] Dalibard, J. and Reynaud, S., J. Physique 44 (1983) 1337.

[34] Goldberger, M. L. and Watson, K. M., "Collision theory " (Wiley) 1964.

[35] Glauber, R. J., in Quantum Optics and Electronics, eds De Witt C., Blandin A. and Cohen-Tannoudji C. (Gordon and Breach) 1965.

[36] Feynman, R. P., Leighton, R. B. and Sands, M., The Feynman Lectures on Physics, vol. III, Ch. 4 (Addison-Wesley, 5th edition) 1975 (Inter-Editions, édition française) 1979.
[37] Stoler, D., Phys. Rev. Lett. 33 (1974) 1397.

[38] Walls, D. F., Nature 280 (1979) 451.

[39] Loudon, R., Rep. Progr. Phys. 43 (1980) 913.

[40] Schubert, M. and Wilhelmi, B., in Progress in Optics. Vol. XVII, ed. Wolf E. (North Holland) 1980, p. 163.

[41] Dalibard, J. and Reynaud, S., same ref. as [18].

[42] Cohen-Tannoudj, C., in Laser Spectroscopy VI, eds Weber H. P. and Lüthy W. (Springer Verlag) 1983.

[43] Cohen-Tannoudj, C., in Frontiers in Laser Spectroscopy, eds Balian R., Haroche S. and Liberman S. (North Holland) 1977.

[44] Carmichael, H. J. and Walls, D. F., J. Phys. B 8 (1975) L77.

[45] Mollow, B. R., Phys. Rev. A 12 (1975) 1919.

[46] Kimble, H. J. and Mandel, L., Phys. Rev. A 13 (1976) 2123.

[47] Kimble, H. J., Dagenais, M. and Mandel, L., Phys. Rev. Lett. 39 (1977) 691.

[48] Dagenais, M. and Mandel, L., Phys. Rev. A 18 (1978) 2217.

[49] Cresser, J. D., Hager, J., Leuchs, G., Rateike, M. and WALther, H., in Dissipative Systems in Quantum Optics, ed. Bonifacio R. (Springer Verlag) 1982.

[50] Mandel, L., Phys. Lett. 89A (1982) 325.

[51] Clauser, J. F., Phys. Rev. D 9 (1974) 853.

[52] Aspect, A., Grangier, P. and Roger, G., Phys. Rev. Lett. 47 (1981) 460.

[53] Burnham, D. C. and Weinberg, D. L., Phys. Rev. Lett. 25 (1970) 84.

[54] Grangier, P. and Aspect, A., Private communication.

[55] Cohen-Tannoudj, C., Cours au Collège de France 1979-80 (unpublished). 